\title{
Identifying the clinical presentations, progression, and sequela of pelvic inflammatory disease through physiological, histological and ultrastructural evaluation of a rat animal model
}

\author{
Linyuan Fan ${ }^{1}$, Zhaohui Liu ${ }^{1}$, Zhan Zhang ${ }^{1}$, Ting Li $^{1}$, Hua Li ${ }^{1}$, Jing Chen ${ }^{1}$, Xiaonan Zong ${ }^{1}$ Xu Zhang ${ }^{2}$, \\ Xi Chen ${ }^{3}$, Huihui Bai ${ }^{4}$, Fengjuan Wang ${ }^{5}$, Chenguang Shang ${ }^{6}$ \\ ${ }^{1}$ Department of Gynecology, Beijing Obstetrics and Gynecology Hospital, Capital Medical University, Beijing Maternal and Child Health Care \\ Hospital, Beijing, China; ${ }^{2}$ Laboratory of Electron Microscopy, Pathological Center, Peking University First Hospital, Beijing, China; ${ }^{3}$ Department of \\ Gynecology, Minimally Invasive Center, Beijing Obstetrics and Gynecology Hospital, Capital Medical University, Beijing Maternal and Child Health \\ Care Hospital, Beijing, China; ${ }^{4}$ Department of Microecological Laboratory, Beijing Obstetrics and Gynecology Hospital, Capital Medical University, \\ Beijing Maternal and Child Health Care Hospital, Beijing, China; ${ }^{5}$ Department of Obstetrics, Beijing Obstetrics and Gynecology Hospital, Capital \\ Medical University, Beijing Maternal and Child Health Care Hospital, Beijing, China; ${ }^{6}$ Department of Gynecologic Oncology, Beijing Obstetrics \\ and Gynecology Hospital, Capital Medical University, Beijing Maternal and Child Health Care Hospital, Beijing, China \\ Contributions: (I) Conception and design: Z Liu, L Fan; (II) Administrative support: L Fan, Z Zhang, T Li, H Li, J Chen, X Zong, X Chen, F Wang, \\ C Shang; (III) Provision of study materials or patients: H Bai; (IV) Collection and assembly of data: L Fan, Z Zhang, X Zhang; (V) Data analysis and \\ interpretation: L Fan, Z Liu; (VI) Manuscript writing: All authors; (VII) Final approval of manuscript: All authors. \\ Correspondence to: Zhaohui Liu. Beijing Obstetrics and Gynecology Hospital, Capital Medical University, No. 251, Yaojiayuan Road, Chaoyang \\ District, Beijing 100026, China. Email: liuzhaohui@ccmu.edu.cn.
}

Background: Patients with pelvic inflammatory disease (PID) are at an increased risk of ectopic pregnancy, infertility, and varying degrees of chronic pelvic pain. The aims of this study were to establish a rat model of PID and characterize its progression in order to assist in the study of pathophysiological mechanisms and to provide animal model for future studies of PID treatments.

Methods: Fifty Sprague-Dawley rats (female, 6-weeks-old) were divided into a model group (n=28) and a control group $(\mathrm{n}=22)$. The rat endometrium was mechanically injured by a needle which moved back and forth 3 times on the endometrial tissue, and a mixed bacterial solution $\left(6 \times 10^{8} \mathrm{CFU}\right)$ of equal concentrations of Escherichia coli and Staphylococcus aureus was injected into both horns of the rat uterus. Physiological characteristics including weight, temperature, blood, and inflammatory factors were compared, and immunohistochemistry and transmission electron microscopy were used to evaluate the progress and sequela of PID.

Results: The model rats experienced acute PID in the first 14 days and exhibited higher body temperatures and decreased body weight. Infection-related factors in the blood were also significantly changed compared with the normal group, with obviously increased serum levels of C-reactive protein (CRP), interferon gamma (IFN- $\gamma$ ), and interleukin-4 (IL-4). Congestion and edema were observed in the uteri of the model rats, followed by infiltration of numerous inflammatory cells and ultrastructural morphology changes. Histological examination of the uterus showed that adhesion initially appeared at approximately 21 days. In addition to the increased collagen fibers biomass, the expression of transforming growth factor-beta 1 (TGF- $\beta 1$ ) was elevated, which might have contributed to pelvic tissue adhesion formation in the PID sequela.

Conclusions: This study clearly described the characteristics and progression of PID in a rat model. The detailed evidence increased our understanding of the pathogenesis and progression of PID and may be useful for future studies of PID treatments.

Keywords: Pelvic inflammatory disease (PID); rat model; tissue adhesion; transforming growth factor-beta 1 (TGF- $\beta 1)$ 
Submitted Jun 28, 2021. Accepted for publication Nov 02, 2021.

doi: 10.21037/atm-21-3345

View this article at: https://dx.doi.org/10.21037/atm-21-3345

\section{Introduction}

Pelvic inflammatory disease (PID) refers to the presence of infection and inflammation in the upper reproductive tract of women, which manifests as endometritis and salpingitis in mild to moderate cases and occasionally as fallopian tube, ovarian abscesses or pelvic peritonitis in severe cases $(1,2)$. According to survey data from the United States Centers for Disease Control and Prevention (CDC), more than 1 million women experience an episode of PID each year, including missed cases of PID (3), and the majority of patients are aged 15-24 years (4). A retrospective crosssectional study revealed that approximately $9 \%$ of women with a chief complaint of abdominal or pelvic pain in outpatient and emergency departments were diagnosed with PID. PID is a major concern because it can result in long-term chronic pelvic pain, ectopic pregnancy, and infertility (5). In a cohort of 100,000 PID women, there were approximately 18,600 cases of chronic pelvic pain, 16,800 cases of infertility, and 8,550 cases of ectopic pregnancy $(6,7)$ Therefore, a detailed description of PID progression is essential to clarifying the mechanism and assisting in diagnosis and treatment of PID (8).

Animal models are used in the study of human diseases because many investigations in biomedical knowledge adopt paradigms that simply cannot be employed ethically in humans. Currently, rat PID models are primarily endometritis or salpingitis models. The rat fallopian tube is quite small, which leads to challenges in performing surgeries and observing experimental outcomes, while the rat uterus possesses specific histological characteristics and accounts for a large portion of the pelvic genitalia. Therefore, a rat model of endometritis is usually established. Based on previous studies, endometritis can be simulated by injecting phenol glue or hydrochloric acid into the uterus (9-11). However, the chemical burns used in these methods are too severe to the endometrium, resulting in animal death. Some researchers implant foreign bodies in the uterus, which stimulates normal tissue to produce a localized inflammatory response. Nevertheless, the pathogenesis of this previous method is not consistent with the natural process of human PID, and the experimental protocol is complex and produces inconsistent results (12). The main cause of PID is an infection of bacteria (including aerobes and anaerobes), mycoplasma, chlamydia, or viruses. To better simulate the pathogenesis of PID, pathogenic microorganisms have been gradually introduced to establish PID animal models in an increasing number of studies (13). Pathological injury may also accelerate or aggravate the development of pelvic inflammation.

Therefore, the aim of this study was to develop a rat model of PID that could elucidate the progression of pelvic inflammation and its sequela in detail. Our findings may serve as further evidence in better understanding the clinical development of pelvic inflammation and as experimental information for drug development and treatment of PID.

We present the following article in accordance with the ARRIVE reporting checklist (available at https://dx.doi. org/10.21037/atm-21-3345).

\section{Methods}

\section{Ethical statement}

This study was an experiment in rat and was conducted in accordance with the Guidelines for the Care and Use of Laboratory Animals and was reviewed and approved by the Animal Ethics Committee of JOINN Laboratories (Beijing, China), with the Ethics Approval Document Number ACU20-1954.

\section{Animals}

Fifty female specific pathogen free (SPF)-grade Sprague Dawley (SD) rats, aged 6 weeks and weighing $200 \pm 20 \mathrm{~g}$ were obtained from Beijing Charles River Laboratory Animal Technology Co. Ltd. (Beijing, China). After passing the quarantine inspection, the rats were raised in a SPFgrade animal room [laboratory animal license number: SYXK(Beijing)2016-0029] at an ambient temperature of $20-26^{\circ} \mathrm{C}$, a relative humidity of $40-70 \%$, and a 12 -h lightdark cycle. This study was conducted in accordance with the Guidelines for the Care and Use of Laboratory Animals (12).

\section{Microorganisms}

Escherichia (E.) coli (ATCC25922) and Staphylococcus (S.) aureus (ATCC25923) were supplied by the Microecology 
Laboratory of Beijing Obstetrics and Gynecology Hospital, Capital Medical University, and the broth solution was purchased from Solarbio Life Sciences Co. Ltd. (Beijing, China). Prior to the experiment, the bacteria were collected in the logarithmic growth phase. The 2 bacterial solutions were combined in a ratio of $1: 1$ to form a mixed bacterial solution with a final concentration of $3 \times 10^{9} \mathrm{CFU} / \mathrm{mL}$.

\section{Animal model}

In accordance with the random digital table, all rats were randomly divided into a sham operation group $(n=22)$ and an experimental model group $(n=28)$. Before surgery, the rats were fasted overnight and then anesthetized with an intraperitoneal injection of $1 \%$ pentobarbital sodium $(30 \mathrm{mg} / \mathrm{kg})$. After achieving a surgical plane of anesthesia, the rats were positioned on the surgical table in a supine position with the hindquarters slightly elevated, which was followed by routine skin preparation and disinfection. A 0.8 to $1-\mathrm{cm}$ incision was made along the midline of the lower abdomen, and the uterus was exposed and securely positioned after laparotomy. Next, a hypodermic needle attached to a $1-\mathrm{mL}$ syringe containing $0.2 \mathrm{~mL}$ of the bacterial suspension was gently inserted into uterine horn in the direction of the fallopian tube. The lack of noticeable resistance with gentle retraction on the syringe plunger indicated that the needle was successfully inserted into the uterine cavity. The needle was moved back and forth 3 times to cause mechanical damage to the endometrial tissue. Then, $0.1 \mathrm{~mL}$ of the bacterial solution was injected into the lumen of the uterine horn. The uterine horn on the other side was subjected to the same protocol. Subsequently, the abdominal cavity was closed, the skin and connective tissues were sutured layer by layer, and the rats were returned into the holding cage. In the sham operation group, after anesthesia, the abdomen of each rat was opened and then immediately closed. The uterus did not undergo any manipulation in the sham group. After surgery, the rats were maintained with free access to food and water.

\section{Observation and sampling}

The behavioral activities, morbidity, breathing, secretions, feces, diet, and water consumption were observed daily for all rats. The rat's body weight and rectal body temperature were measured, and a blood sample was collected from the tail vein for routine blood tests every 2 days between post operative days 0 and 14 . Samples were collected at 3 ,
$7,14,21,42$, and 60 days after surgery. Under isoflurane anesthesia, the eyes of each rat were removed, and $2 \mathrm{~mL}$ of blood were collected. The abdominal cavity was opened, and the pelvic organs, including the uterus and fallopian tubes, were collected.

\section{Enzyme-linked immunosorbent assay}

According to the manufacturer's instructions, the experimental procedures were primarily as follows. Serum $(100 \mu \mathrm{L})$ was added to each coated reaction well, sealed with a plate membrane, and incubated at $37^{\circ} \mathrm{C}$ for 1-2 h. After a washing with phosphate-buffered saline (PBS), biotinylated antibody was added and was followed by incubation for $1 \mathrm{~h}$. After another wash with PBS, the chromogenic substrate was added, and then $100 \mu \mathrm{L}$ of $2 \mathrm{M}$ sulfuric acid was added into each reaction well to terminate the reaction. Subsequently, the optical density $(\mathrm{OD})$ value at $450 \mathrm{~nm}$ was measured using a microplate reader, and the concentration of the sample was calculated based on a standard curve.

\section{Hematoxylin and eosin staining and analysis}

Tissues were fixed with $10 \%$ neutral formalin solution and subsequently dehydrated, cleared using xylene, embedded in paraffin, and sectioned to a 4- $\mu$ m thickness. Subsequently, the paraffin was removed using xylene, rehydrated using a graded series of alcohols, and finally underwent routine hematoxylin and eosin (HE) staining. Tissue morphology and inflammation were observed under amicroscope (Olympus, Tokyo, Japan). Inflammatory cells were identified, including lymphocytes, eosinophils, neutrophils, and plasma cells. The degree of inflammatory infiltration was evaluated based on the number of inflammatory cells present. The infiltration of inflammatory cells into the uterine mucosa and muscular layer was observed to comprehensively assess the degree of inflammation of the uterine tissue.

\section{Immunobistochemistry}

The tissue sections were prepared as previous described. The sections antigens were first repaired by heating and then sealed with a serum within cubation overnight at $4{ }^{\circ} \mathrm{C}$ with the transforming growth factor-beta 1 (TGF- $\beta 1)$ polyclonal antibody $(1: 100$; cat no. 21898 1-AP; Proteintech, Wuhan, China). Negative control 


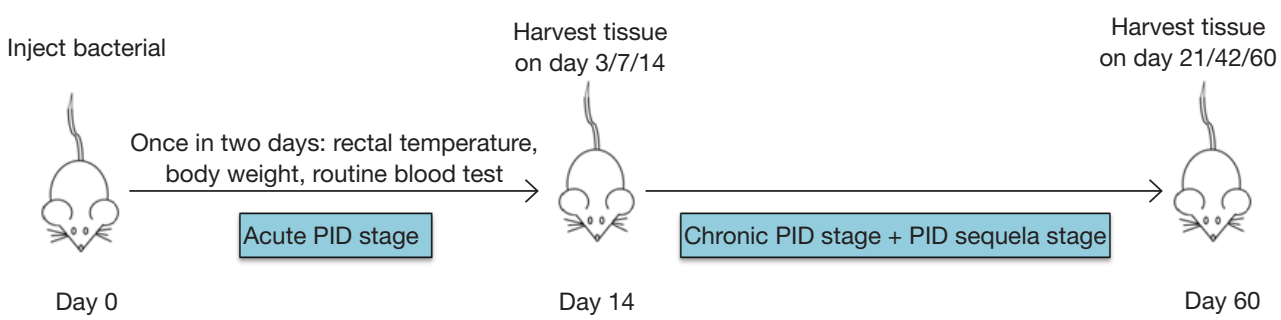

Figure 1 Schematic diagram of the experimental and sample collection.

sections were incubated with PBS instead of the primary antibody. Next, biotin-conjugated anti-rabbit immunoglobin (IgG) was incubated with sections at room temperature for $30 \mathrm{~min}$. Sections were then incubated with horseradish peroxidase-conjugated anti-rabbit IgG (Beijing Zhongshan Golden Bridge Biotechnology, Beijing, China), and immunoreactivity was developed using 3,30-diaminobenzidine (DAB) substrate system staining.

\section{Masson's trichrome staining}

Masson's trichrome staining was conducted according to the instructions of Masson Stain Kit (cat no.60532ES58, YEASEN Biotechnology, Shanghai, China). Briefly, prepared tissue slides were stained in Weigert's hematoxylin for $60 \mathrm{~s}$ and then washed with tap water for 30s. Next, the slides were stained in Biebrich scarlet-acid fuchsin solution for $60 \mathrm{~s}$, rinsed in distilled water, incubated in phosphotungstic-phosphomolybdic acid for 6 minutes, dyed with aniline blue for 5 minutes, and washed with anhydrous ethanol. Finally, the slides were rinsed in distilled water, dehydrated, and mounted. Semiquantitative analysis was performed by an experienced pathologist.

\section{Transmission electron microscopy}

Vaginal tissues were removed and fixed in $3 \%$ glutaraldehyde and $1 \%$ osmium tetroxide at least $24 \mathrm{~h}$ at $4{ }^{\circ} \mathrm{C}$ as previously reported (12). Tissues were then dehydrated and embedded, and then cut into $70-\mathrm{nm}$ thick slides, which were embedded in PON812 epoxy resin (SPI, West Chester, PA, USA) and observed under transmission electron microscopy (JEM1230, JEOL Co., Hitachi Ltd., Tokyo, Japan). The ultrastructure morphological features within the epithelium, such as endoplasmic reticula and mitochondria, were observed and photographed independently by 2 investigators.

\section{Statistical analysis}

GraphPad Prism 5.0 (GraphPad Software Inc., San Diego, CA, USA) and SPSS Statistics 22.0 software (IBM Corp., Armonk, NY, USA) were used for plotting and analysis of all data. Results are shown as mean \pm standard deviation. Data conforming to normal distribution were evaluated using a 2-tailed unpaired Student's $t$-test; otherwise, the Mann-Whitney $U$ statistical test was used. A P value of $<0.05$ was considered statistically significant.

\section{Results}

Pronounced inflammatory responses occurred 14 days after the establishment of the PID model

The general experimental protocol was as follows (Figure 1). The day of establishment of the rat model through surgery was considered to be day (D) 0 . Based on a comprehensive evaluation of the clinical characteristics exhibited by the rats, D0-14 were considered to be the acute PID stage, while D14-60 were considered to be the chronic and sequela stage. Every 2 days during the acute PID stage, the rectal temperature and body weight of the rats were measured, and $200 \mu \mathrm{L}$ of blood was used for routine blood tests $(\mathrm{N}=15)$. Every 3 rats were euthanized on $\mathrm{D} 3,7,14$, 21, 42, and 60, and the PID symptoms and locations of adhesions were observed and followed by tissue sampling.

A systemic inflammatory response often accompanies acute PID. To evaluate the occurrence of acute PID, the body temperatures (represented by the rectal temperature) of the rats were compared (Figure 2A). On D2 after surgery, the body temperature increased in both the model and sham groups, but the increase in body temperature in the model group was more pronounced than that in the sham group. From D4 to D10, the rectal temperatures in the model group were significantly higher than those in the sham group, indicating that the pelvic inflammation had spread 

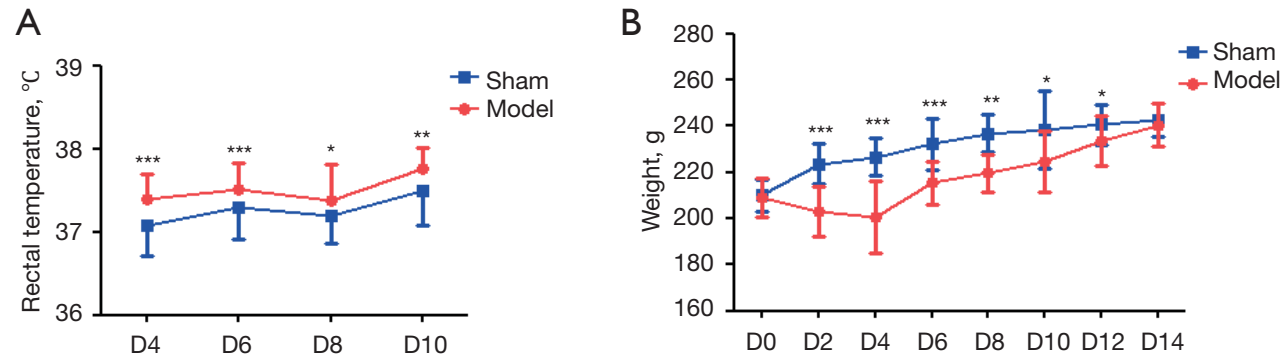

Figure 2 Changes of rectal temperature (A) and weight (B) of rat in acute PID period. The sham group was the control and model group was the PID group. All data were expressed as the mean $\pm \mathrm{SD}(\mathrm{n}=15) .{ }^{*} \mathrm{P}<0.05,{ }^{* *} \mathrm{P}<0.01,{ }^{* * *} \mathrm{P}<0.001$. PID, pelvic inflammatory disease; SD, standard deviation.

to the entire body of the experimental rats postoperatively, resulting in a high fever and systemic inflammatory response.

There also was a discernible difference in body weights between the sham and model groups from D0-12 (Figure $2 \mathrm{~B}$ ). Although there was a definite increase in body temperature, the body weights of the rats increased slowly. In the model group, specifically, the mean body weight decreased markedly between D0 $(213.5 \pm 8.67)$ and D2 $(200.7 \pm 15.52)$, and then to $203.2 \pm 10.85$ on D4. Subsequently, the body weights of the rats in the model group increased but were still dramatically lower than those in the sham operation group between D6-12. It was not until D14 that the body weights in the model and the sham operation groups were comparable.

\section{Changes in infection indices in the blood of acute PID rats}

Acute infection occurred in rats on the second day after surgery, which lasted approximately 2 weeks, and then the rats entered the chronic inflammation stage. By analyzing changes in the white blood cell (WBC) profile, a transient elevation in leukocyte levels was observed on D 2 in the sham operation group due to the laparotomy (Figure $3 A$ ). In the model group, the leukocyte levels declined temporarily on D2 due to severe inflammation and then began to rise gradually. The WBC levels in the model group were markedly higher than those in the sham operation group on D6 and D8, but declined on D10, and returned to normal on D14. A similar situation was observed for platelet (PLT) counts (Figure $3 B$ ). The $\log _{10} \mathrm{PLT}$ in the model group decreased considerably on D2-4 and then rose. The $\log _{10} \mathrm{PLT}$ in the model group was higher than that in the sham operation group until D14. Also, the neutrophil ratio (NE\%) in the model group increased continuously between D0-8, peaking on D8, and was significantly higher than that in the sham operation group (Figure 3C). Subsequently, the NE\% decreased, with almost no difference between the 2 groups on D14. In the model group, the monocyte ratio (MO\%) increased sharply on D2 and then decreased slightly but was still appreciably higher than that in the sham operation group until D10 (Figure 3D). However, the lymphocyte ratio (LY\%) in the model group was dramatically lower than that in the sham operation group between D0-14 (Figure 3E). Due to the severe inflammatory response and decreased food intake, in contrast with levels observed on D0, the hemoglobin (HGB) in the model group decreased prominently from D4 $(143.00 \pm 8.00)$ and reached the lowest levels on D8 $(133.00 \pm 5.00)$ (Figure $3 F)$. Although the HGB in the sham operation group declined somewhat (possibly related to the laparotomy), the HGB was still lower in the model group compared to that in the sham operation group until D14. The HGB did not return to preoperative levels in either group.

Enzyme-linked immunosorbent assay (ELISA) was used to assess the expression of serum inflammatory factors, including C-reactive protein (CRP), interferon gamma (IFN- $\gamma$ ), and interleukin-4 (IL-4). The results indicated that CRP was significantly higher in the model group than in the sham operation group on D3, indicating that there was a severe inflammatory response in the model group (Figure $4 A$ ). By D7, the inflammatory response caused by the laparotomy in the sham operation group had disappeared, and the CRP level had decreased. In the model group, the degree of the acute inflammation response was reduced, so the CRP level declined, but it was still significantly higher compared to that in the sham operation group.

IFN- $\gamma$ is a critical inflammatory factor. The expression of IFN- $\gamma$ in the model group increased substantially on D3 (Figure 4B). It was slightly lower on D7 compared to D3 but was significantly higher than in the sham operation group. 


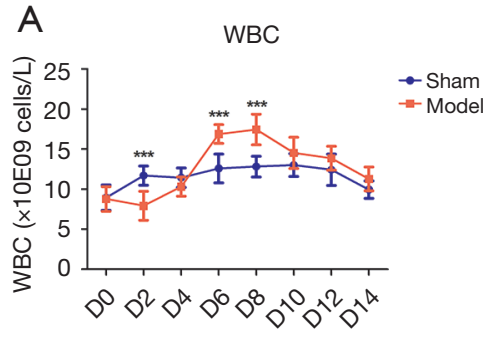

D

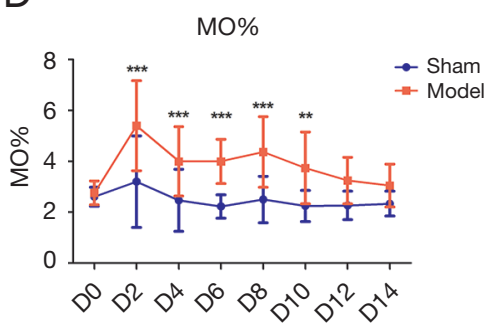

B

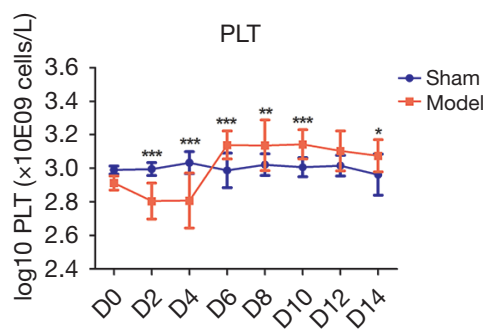

$E$

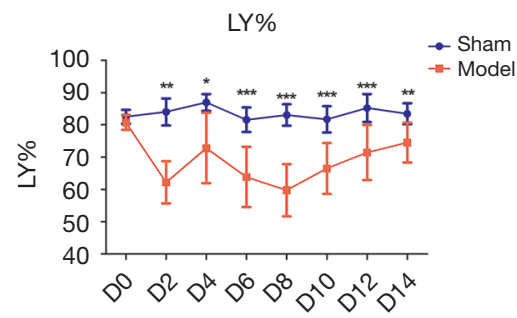

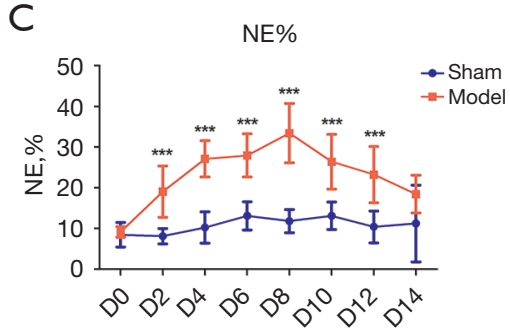

F

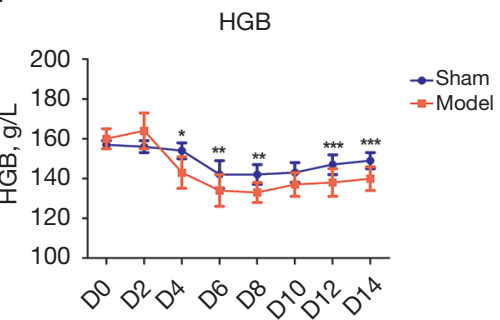

Figure 3 Changes in infection indices in the blood of acute PID rats. The routine blood test was performed to evaluate the level of $\mathrm{WBC}$ (A), PLT (B), NE\% (C), MO\% (D), LY\% (E) and HGB (F). All data were expressed as the mean $\pm \mathrm{SD}(\mathrm{n}=15) .{ }^{*} \mathrm{P}<0.05,{ }^{* *} \mathrm{P}<0.01,{ }^{* * *} \mathrm{P}<0.001$. WBC, white blood cells; PLT, platelet count; NE\%, neutrophil ratio; MO\%, monocyte ratio; LY\%, lymphocyte ratio; HGB, hemoglobin; PID, pelvic inflammatory disease; SD, standard deviation.
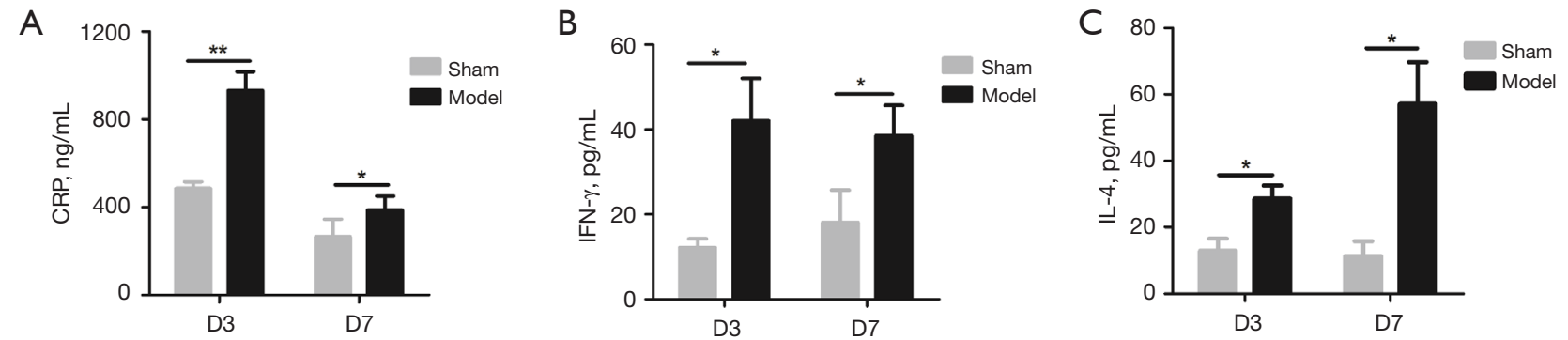

Figure 4 The expression of serum inflammatory factors CRP (A), IFN- $\gamma$ (B) and IL-4 (C) were tested by ELISA analysis on D3 and D7. All data were expressed as the mean $\pm \mathrm{SD}(\mathrm{n}=15)$. ${ }^{*} \mathrm{P}<0.05,{ }^{* *} \mathrm{P}<0.01$. ELISA, enzyme-linked immunosorbent assay; SD, standard deviation; CRP, C-reactive protein; IFN- $\gamma$, interferon gamma; IL-4, interleukin-4.

IL-4 is an essential humoral immune factor. On D3, the level of IL-4 in the model group was notably higher than that in the sham operation group (Figure 4C). Moreover, on D7, the level of IL-4 in the model group continued to increase, implying that the inflammation was aggravated in the model group and accompanied by more extensive antibody responses.

\section{A noticeable tissue inflammatory response appeared in acute PID rats}

After dissection, the morphology of the pelvic tissues was first assessed grossly. In the model group, congestion and edema were evident in the uterine tissue, which included welling and thickening of the uterus as well as the presence of a localized sticky and pus-like substance (Figure 5A). Edema and swelling were observed in the vaginal tissues, along with distinct swelling in the uterus, fallopian tubes, and ovarian tissue in the model group. Moreover, the color of the uterus was considerably darker due to hyperemia and congestion that had formed and filled the uterine cavity.

HE staining was used to assess the degree of inflammation in the uterine tissue on D4 and D14 (Figure 5B). With routine HE staining, lymphocytes appeared small and round, with a very high ratio of nucleus to cytoplasm. The lymphocyte nucleus stains dark blue-purple. Eosinophils 
A

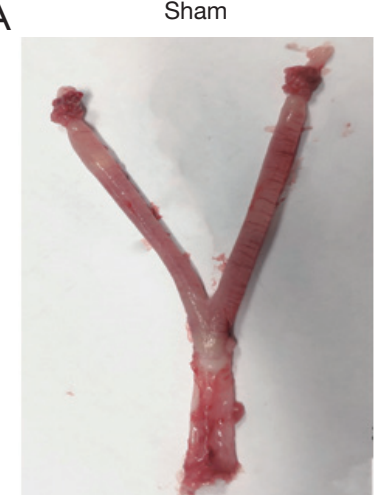

B

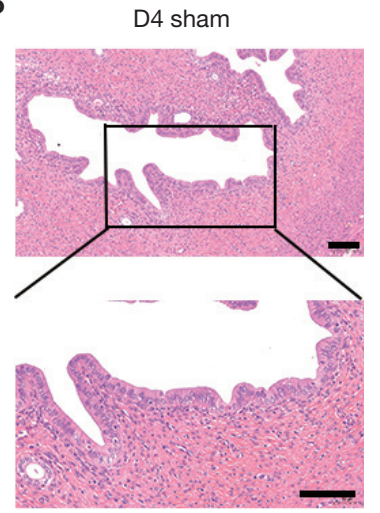

Model 1

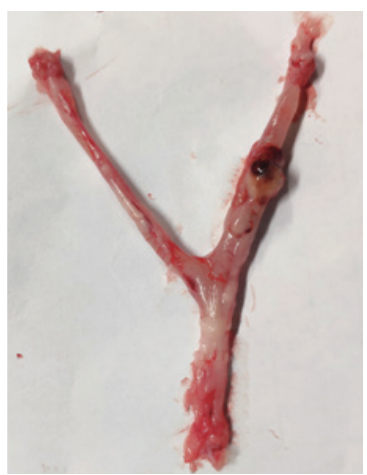

D4 model

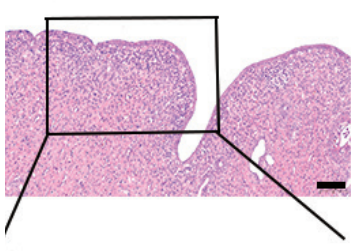

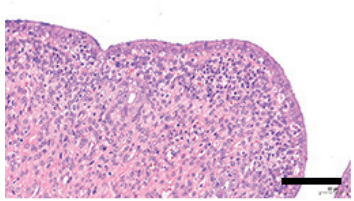

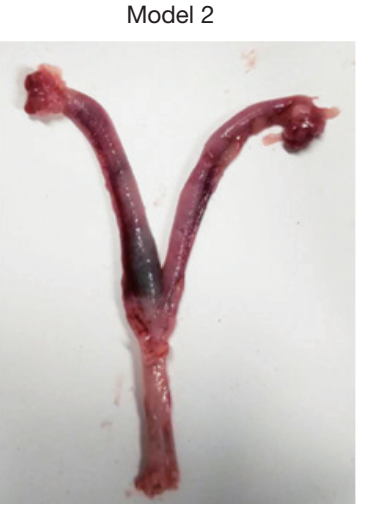

C

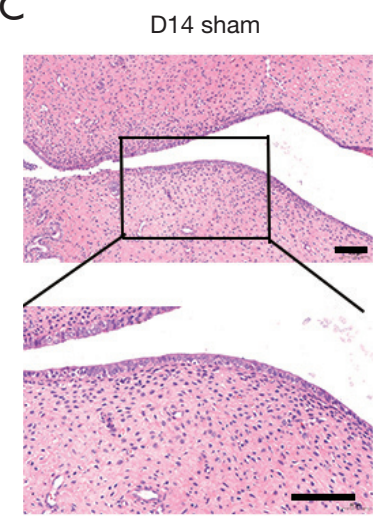

Model 3

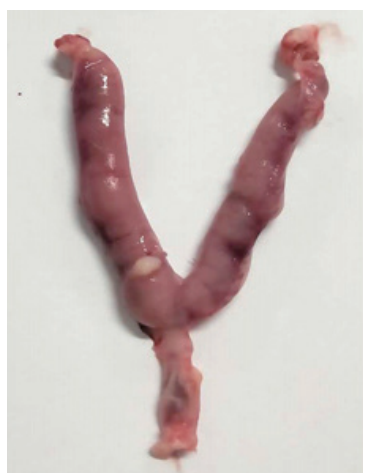

D14 model

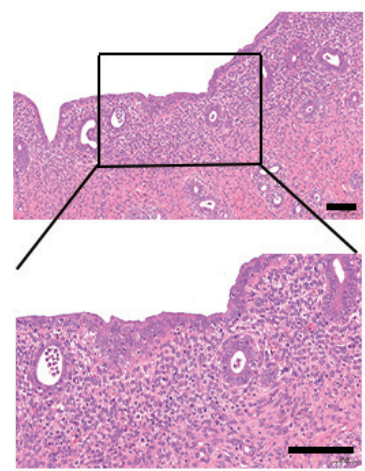

Figure 5 A noticeable tissue inflammatory response appeared in acute PID rats. Morphology of the pelvic tissue was assessed after dissection on D14 (A). Dissection congestion were evident in the uterine tissue (A, model 1), hyperemia leads considerable darker color of the uterus, allopian tubes, and ovarian tissue (A, model 2), and edema formed and filled the uterine cavity (A, model 3). The degree of inflammation in the uterine tissue was assessed by $\mathrm{HE}$ staining analyses (B,C). The infiltration of massive numbers of lymphocytes, neutrophils, and plasma cells were observed and concentrated in the mucosal layer (B, D4model). On D14, some epithelial cells appeared degenerated and undergoing necrosis, inflammatory cells extended to the muscular layer (C, D14 model). Scale bars: $100 \mu \mathrm{m}$. PID, pelvic inflammatory disease; HE, hematoxylin and eosin.

exhibit a bilobed nucleus, and neutrophils generally have a nucleus with 3 to 5 lobes. Plasma cells present an eccentric nucleus that is darkly stained with a mottled appearance resembling a spoked wheel when visualized at high magnification. In the sham operation group, the structure of the uterine wall was well defined, the epithelial tissue and glands were intact, no inflammatory cell infiltration was seen, and the muscle fibers were arranged normally. On D4, the infiltration of massive numbers of lymphocytes, neutrophils, and plasma cells were observed in tissues from the model group. Suppurative foci appeared in the mucous membranes, and the area of inflammatory infiltration was primarily concentrated in the mucosal layer. On D14, the structure of the uterine wall was disorganized in the model group. In addition, proliferation and changes in the arrangement of the columnar epithelial cells were observed, with some epithelial cells that appeared to be degenerated and undergoing necrosis. Moreover, the lymphocytes, neutrophils, and plasma cells had infiltrated through the mucosa and submucosa to the muscular layer (Figure 5C). The HE staining results of all uteri were evaluated based on the observed degree of inflammation and the extent of inflammatory cell infiltration.

The uterus epithelial cells, their junctional complexes, and mitochondria were examined by transmission electron microscopy. The uterine mucosal epithelial cells of control rat exhibited a normal arrangement, and the outer mitochondrial membrane and crests of the mitochondria were intact. Microvilli, which are regarded as an early morphological indicator of cell damage, are formed by extensions of the cell membrane and are covered by glycocalyx. In the sham group, the epithelial cells had 
A

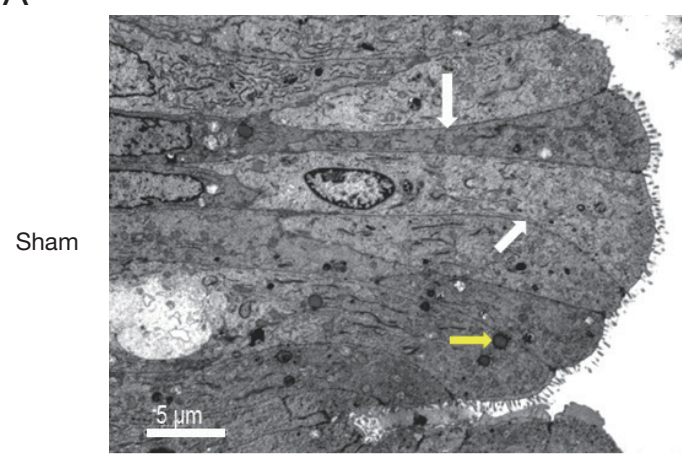

B

Model

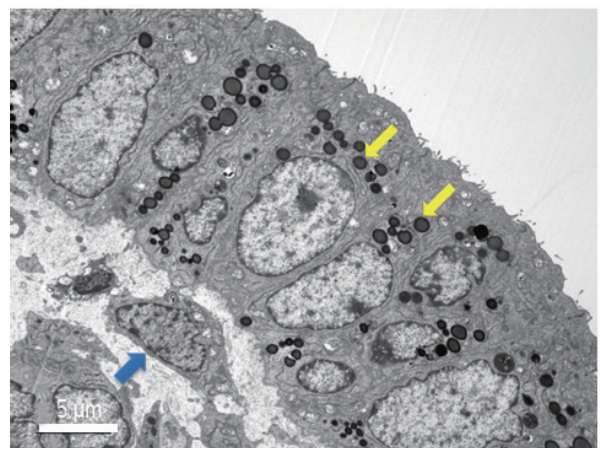

×30000 magnification
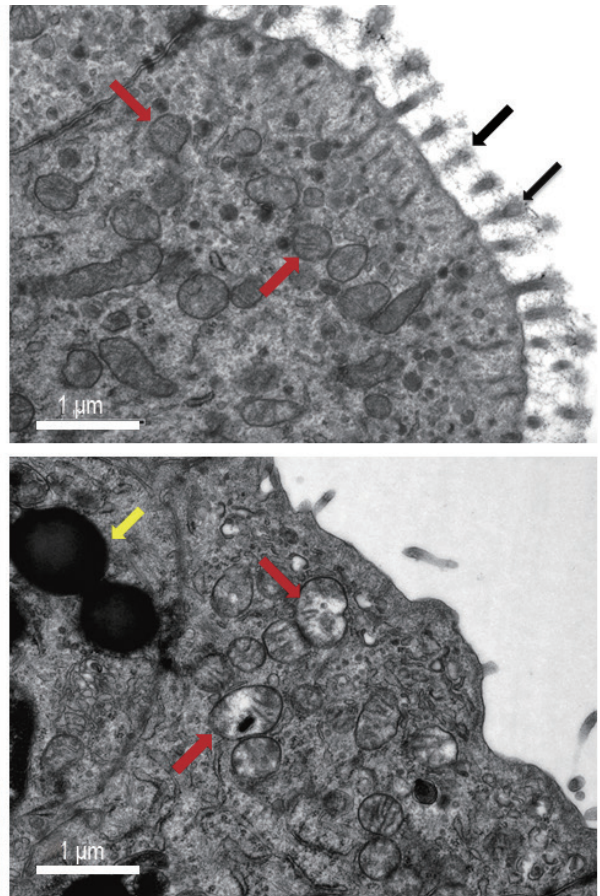

Figure 6 Ultrastructural morphology changes in the uterus epithelium of rats on D14 was observed by transmission electron microscopy. The sham group showed normal uterus morphology with an obvious intercellular adhesion (white arrow) and few lipid drop (yellow arrow) in simple columnar epithelium cells, which has normal mitochondria morphology (red arrow) and rich intact microvilli (black arrow) (A). The uterus mucosa of model group were more infiltrated by mononuclear inflammatory cells (blue arrow) and numerous lipid drop. The microvilli of the model group were disrupted and glycocalyx was reduced sharply or even disappeared. The mitochondria were observed with ruptured membranes, swelling, and low cell matrix density (B).

numerous intact finger-like microvilli and rich glycocalyx (Figure 6A). However, the uterus mucosa of model group was more infiltrated by mononuclear inflammatory cells and numerous lipid drops. Furthermore, compared with control, the microvilli of the model group were often disrupted, and glycocalyx was reduced sharply or had even disappeared. Intracellularly, the adhesion of epithelial cells was often reduced and destroyed, and mitochondria were observed with ruptured membranes, swelling, and low cell matrix density (Figure 6B). These observations indicated that inflammation induced a partial destruction of the uterine mucosa.

\section{Pelvic tissue adbesion of rats in chronic and PID sequela stages}

The noticeable tissue inflammatory response gradually subsided after 14 days, and then PID entered the chronic inflammatory phase. Meanwhile, on D 21, the adhesion of pelvic tissue was observed, which is the typical characteristic of the PID sequela phase. The morphology of the pelvic tissues was assessed after dissection. In the model group, adhesions appeared around the uterine tissues, which were closely related to the surrounding pelvic tissue (Figure $7 A$ ). In the PID sequela stage, the endometrial epithelial cells exhibited normal morphology, and the shape and size of the uterine cavity were normal in the sham operation group (Figure 7B). However, in the model group, the uterus was tortuous and swollen, local adhesions were observed in the endometrium, the uterine cavity was reduced, and infiltration of inflammatory cells was observed at the adhesion sites. On D60, the uterine adhesions were increased overall. The endometrial adhesions were extensive, and the infiltration of lymphocytes and macrophages could be seen throughout the entire tissue sample (Figure 7C). 
A

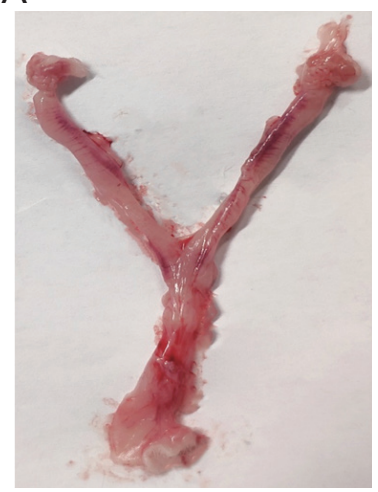

B

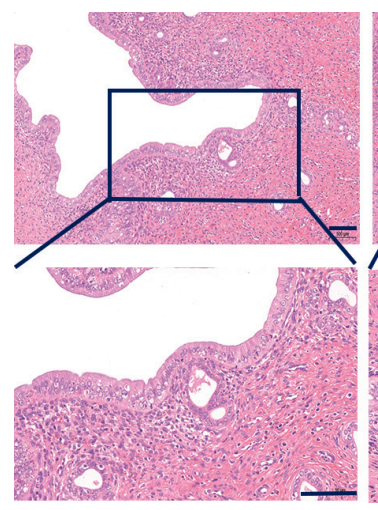

Model 1

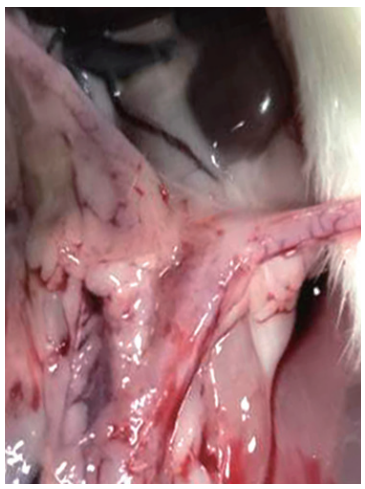

D21 model

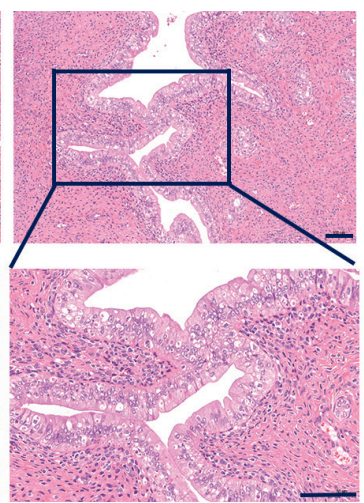

Model 2

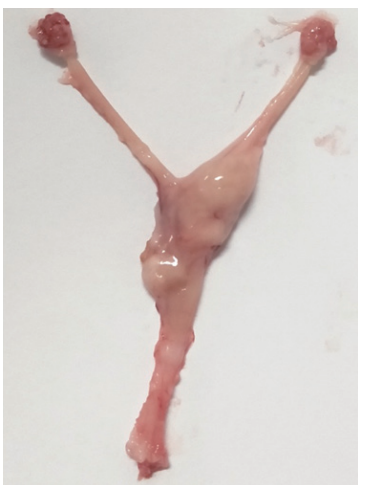

C

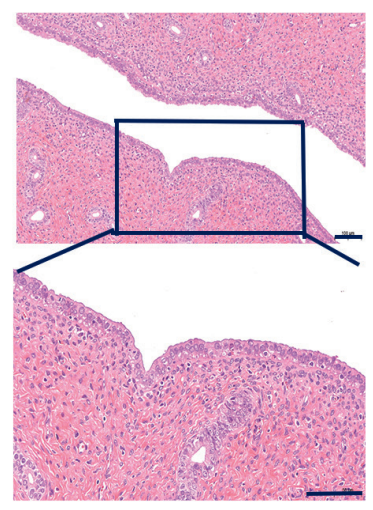

Model 3

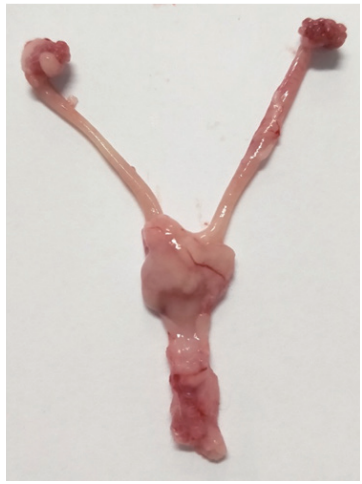

D60 model

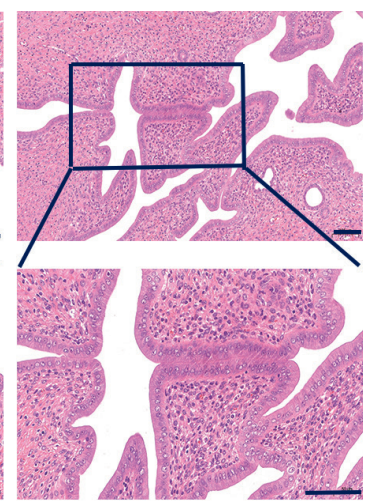

Figure 7 The pelvic tissue adhesion of PID sequela rats on D21. The morphology of the pelvic tissue was assessed after dissection. In the model group, adhesion appeared around the uterine tissue, which was closely related to the surrounding pelvic tissue (A, model 1 , model 2, model 3). The adhesion degree of uterine tissue was evaluated after HE staining (B,C). The uterine cavity was reduced and endometrial adhesions which areas had filtration of inflammatory cells were observed in the model group (B). On D 60, overall, the uterine adhesions were extensive, and the infiltration of lymphocytes and macrophages could be seen throughout the entire tissue samples (C). Scale bars: 100 $\mu \mathrm{m}$. PID, pelvic inflammatory disease; HE, hematoxylin and eosin.

\section{Potential contribution of TGF- $\beta 1$ and collagen fibers to the formation of uterine adhesions}

TGF- $\beta 1$ is one of the essential profibrogenic factors related to inflammation. On D21 and D60, almost no positive TGF- $\beta 1$ signal was observed in the uterine tissue of the sham operation group. However, TGF- $\beta 1$ staining in uterine tissue in the model group was considerably more intense, and the TGF- $\beta 1$ expression level was increased. Moreover, epithelial cells of the uterine mucous layer with or without adhesions were stained brown, indicating that TGF- $\beta 1$ was highly expressed, which implied that the endometrium in the model group might have grown increasingly susceptible to adhesion due to the inflammatory response. On D60 in the model group, the adhesions of the uterine tissues were increased, and TGF- $\beta 1$ expression in the uterine tissues was higher than that in the control group (Figure 8A).

Masson's trichrome staining was used on the uterine tissues obtained on D42. In the sham operation group, the cells in the mucosa, submucosa, muscle layer, and serosa were stained dark red, while the collagen fibers in the endometrial stroma were stained blue. In the model group, the collagen fibers in the uterine tissues were more darkly stained in general (Figure 8B). The serosa was stained blue, and the overall areas of tissue that stained blue were markedly increased (Figure 8C). The collagen fiber area rate and average optical density (AOD) of model group were both significantly increased compared to those in the model group $(\mathrm{P}<0.05)$, suggesting that the number of collagen fibers in the uterine tissues was increased on D42 and that the serosa layer was extremely prone to adhesions. 


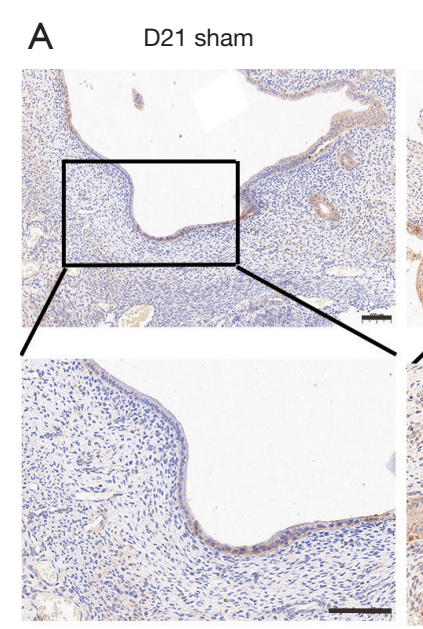

B

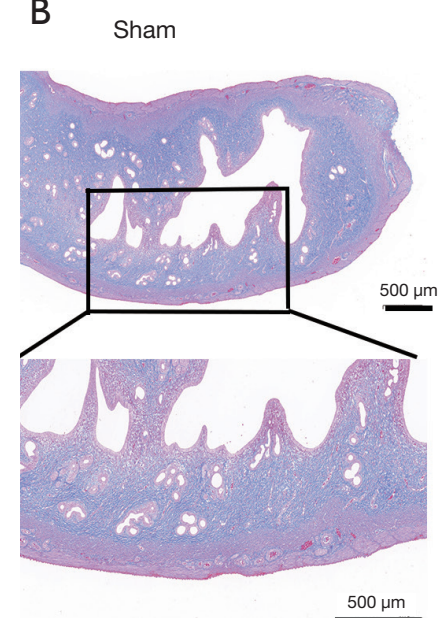

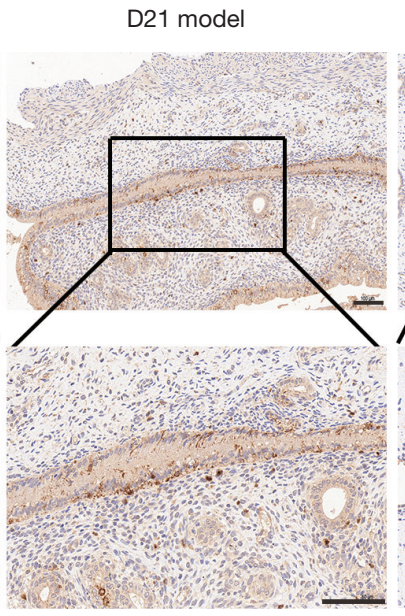

Model

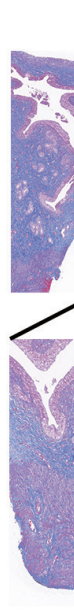

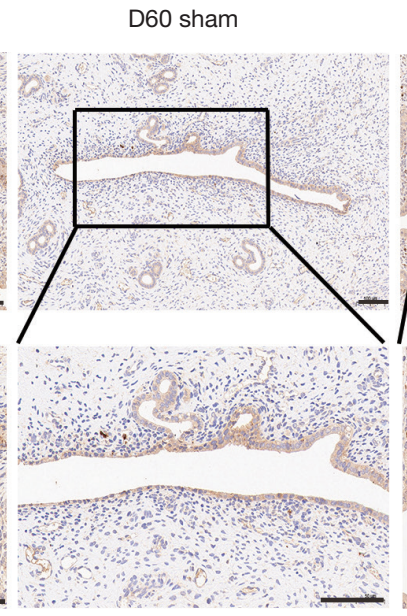

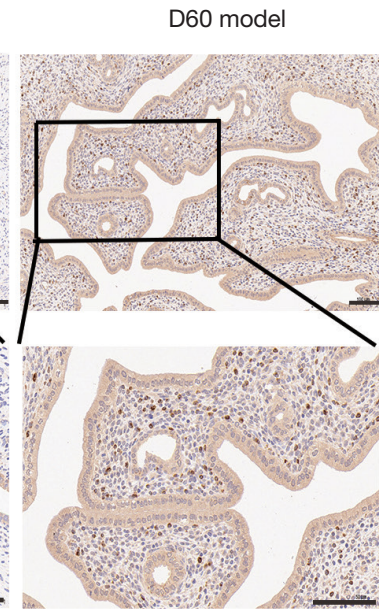

C
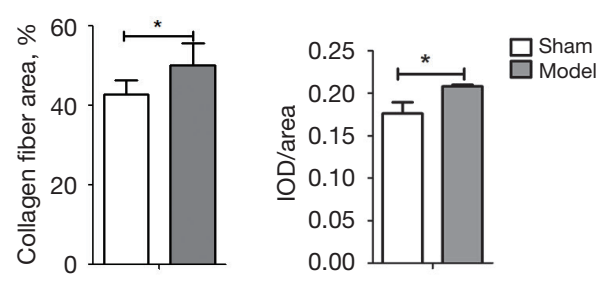

Figure 8 Changes of the expression of TGF- $\beta 1$ and collagen fibers in adherent uterine tissue. The expression of TGF- $\beta 1$ in adhesion uterine tissue of PID sequela (D21, D60) was detected by immunohistochemistry (A), Scale bars: $100 \mu \mathrm{m}$. The collagen fibers expression in uterine tissue was detected by Masson's trichrome staining and was stained blue (B). Image-Pro Plus 6.0 analysis software was used for Masson's trichrome staining analysis and pixel area was used as the standard unit (C). Each group choose three tissues, and each tissue section choose five visual fields. IOD, integrated optical density; average optical density (AOD) = IOD/Area(collagen positive pixel area); collagen fiber area $(\%)=$ collagen positive pixel area/tissue pixel area $\times 100 .{ }^{*} \mathrm{P}<0.05$. TGF- $\beta 1$, transforming growth factor-beta 1; PID, pelvic inflammatory disease.

\section{Discussion}

The establishment of an animal model that mimics the natural process of PID occurrence in human females is essential to investigating the pathogenesis of PID. In this study, a rat model of endometritis was initially established. Inflammation then ascended through the endometrium to the fallopian tube and ovary, ultimately developing into PID. PID is induced by multiple pathogens, including the common exogenous pathogens, Neisseria (N.) gonorrboeae and Chlamydia (C.) trachomatis. N. gonorrboeae, the first PID pathogen to be reported, is more likely to result in severe symptoms than other pathogens (14). However, due to universal screening and treatment for gonorrhea in many countries, the incidence rate of gonorrhea has decreased, and its role in PID has been reduced. C. trachomatis and Mycoplasma (M.) genitalium often cause asymptomatic subclinical infections, which manifest as chronic pelvic pain or infertility several years after the initial infection. Thus, a longer time is likely needed to establish an animal model of PID using chlamydia (15). 
Recently, numerous studies have suggested using mixed infections of endogenous pathogens, such as anaerobes, Gram-negative Enterobacter, Streptococci, and Gardnerella vaginalis related to bacterial vaginosis, which might play an important role in the pathogenesis of PID (16). Some researchers simulated the ascending infection of PID in the reproductive tract by placing sponges containing bacteria in the vagina. However, due to the barrier function of the cervical canal, the aseptic upper reproductive tract is difficult to infect with microorganisms found in the vaginal ecosystem. The lower reproductive tract bacteria can induce PID in some women but not in others, and the reason for this remains unclear. Possible explanations include genetic variations in the immune response, estrogen's influence on cervical mucus viscosity, or the load of potentially pathogenic bacteria (17).

Based on previous studies, the PID model used in this study was established using a direct intrauterine injection of pathogenic microorganisms. Both Staphylococcus aureus and E. coli are pathogenic microorganisms associated with PID. The former is a common pathogen of postpartum, post-surgery, and genital inflammation, as well as wound infections in women. The latter normally resides in the intestinal tract and vagina and generally does not cause disease. However, E. coli can induce severe infection when the body is debilitated and the reproductive tract is injured. In this study, reproductive tract injury was simulated through mechanical damage to the endometrium, and the above 2 bacteria were selected as the pathogenic microorganisms. Based on preliminary exploration, it was determined that the optimum concentration for the bacteria was $3 \times 10^{9} \mathrm{CFU} / \mathrm{mL}$, and the rat model of PID was successfully established.

Patients with acute PID often have intense episodes of lower abdominal or pelvic pain, pelvic organ tenderness, and reproductive tract inflammation, with systemic inflammation and fever occurring in severe cases (18). After the bacteria were injected into the rat uterus, distinct PID symptoms were observed, including pelvic tenderness, elevated body temperature, reduced activity, weight loss due to decreased food intake, massive uterine effusion, abscess foci, and fallopian tube and ovarian tissue swelling and hyperemia. Moreover, pathological examinations revealed suppurative foci in the uterine mucosa and infiltration of inflammatory cells into the uterine myometrium. Considering the daily behaviors of the experimental rats and changes in the serum inflammatory factors, D0 to D14 were determined to be the acute stage of rat PID. In the acute stage of PID, the rats not only exhibited high fever, but also exhibited pronounced abnormalities in multiple infectionrelated components in serum, including WBC, PLT, $\mathrm{NE} \%, \mathrm{MO} \%$, and $\mathrm{LY} \%$, suggesting that these are notable symptoms of infection in rats. Between postoperative D0 to D4, WBC and PLT declined transiently, which might have been related to the severe inflammation associated with the early stages of PID, and CRP expression in the blood was highest at this time. Due to the inflammation, the HGB of the rats decreased dramatically during D0-8 (the acute stage of PID) and then leveled off. These observations indicate that this model provided an excellent simulation of the clinical conditions seen in patients with acute PID.

The inflammatory response represents a vital component of acute PID. When the body is stimulated by a range of external pathogens, many inflammation-related factors, such as IFN- $\gamma$, IL- $1 \beta$, and IL-4, are released. IFN- $\gamma$, a cytokine produced by $\mathrm{T}$ helper type 1 (Th1) cells, can mediate the cellular immune function together with IL-2 when infections with viruses and pathogenic microorganisms occur, leading to an inflammatory response and tissue injury (19). The expression of IFN- $\gamma$ was significantly elevated on D3 following establishment of the PID model, suggesting that the differentiation of Th0 cells into Th1 cells was enhanced and the cellular immune response was activated. IL-4, which is produced by activated Th2 cells, promotes $\mathrm{T}$ cell growth and Th2 cell differentiation and enhances the antibody response in vivo. IL-4 also suppresses the inflammatory response by inhibiting the release of TNF- $\alpha$ and IL- $1 \beta$ by mononuclear macrophages (20). In this study, it was found that in the early stage of acute PID, IL-4 expression was not dramatically increased. However, with the progression of PID, the IL-4 expression levels rose sharply on D7, implying that the antibody immune response was activated on D7. According to a previous study, negative feedback regulation takes place between cytokines secreted by Th1 and Th2, and the regulation between the Th1 and Th2 cytokines is involved in the inflammatory process and is associated with the progression of PID (21). After a comparison of the data for IFN- $\gamma$ and $\mathrm{IL}-4$, we found that in the early stage of PID, the expression of the proinflammatory response factor, IFN- $\gamma$, was at its peak, while IL-4 expression was low. Subsequently, an imbalance occurred between Th1 and Th2, and the IL-4 expression was elevated, which inhibited the level of IFN- $\gamma$. This observation was consistent with a previous report in which IFN- $\gamma$ (secreted by Th1 cells) was reported to inhibit the production of Th2 cytokines, while IL-4 (secreted 
by Th2 cells) was reported to downregulate the effects produced by Th1 cytokines (22). Another study suggested that IL-4 could facilitate the proliferation of fibroblasts, thereby aggravating tissue injury and scar tissue formation, which may be associated with the formation of PID sequela (23).

Endometrial fibrosis is the major pathological factor underlying uterine adhesions, and TGF- $\beta 1$ is a critical profibrogenic factor that plays a vital role in cell growth and differentiation, inflammation, tissue repair, and immune function (24). In a previous study, the expression levels of TGF- $\beta 1$ and its receptor were increased significantly in various types of cells at the sites of fibrous adhesions after abdominal wall injury (25). In the present study, it was observed that in the early stage of PID sequela, continuous inflammatory stimulation led to partial adhesions of the endometrium and increased secretion of TGF- $\beta 1$ by endometrial mucosal epithelial cells, which might have increased the expression of collagen fibers. This finding is consistent with a clinical study that reported TGF- $\beta 1$ expression to be increased in the adhered tissues of patients with uterine adhesions (26). TGF- $\beta 1$ stimulates stromal cell growth and excessive proliferation and differentiation of fibroblasts, resulting in increased collagen synthesis and extracellular matrix deposition, thus promoting fibrous scar tissue formation $(27,28)$.

\section{Conclusions}

The rat model of PID and its sequela used in this study was successfully established using mechanical trauma to the endometrium and coinfection with $E$. coli and $S$. aureus in the injured rat endometrium. The characteristics of the occurrence and development of PID and the interrelationships between the major factors were described comprehensively. These results provide excellent references for clinical research on the occurrence and progression of PID. Also, we observed that the expression of TGF- $\beta 1$ and collagen fibers was increased in the adhesion tissue during the sequela of PID, suggesting that TGF- $\beta 1$ might promote tissue adhesion during the PID sequela.

\section{Acknowledgments}

Funding: This work was supported by a grant from the National Natural Science Foundation of China (81771530 and 81901450).

\section{Footnote}

Reporting Checklist: The authors have completed the ARRIVE reporting checklist. Available at https://dx.doi. org/10.21037/atm-21-3345

Data Sharing Statement: Available at https://dx.doi. org/10.21037/atm-21-3345

Conflicts of Interest: All authors have completed the ICMJE uniform disclosure form (available at https://dx.doi. org/10.21037/atm-21-3345). The authors have no conflicts of interest to declare.

Ethical Statement: The authors are accountable for all aspects of the work in ensuring that questions related to the accuracy or integrity of any part of the work are appropriately investigated and resolved. Experiments were performed under a project license (No. ACU201954) granted by the Animal Ethics Committee of JOINN Laboratories (Beijing, China), in compliance with Care and Use of Laboratory Animals national guidelines for the care and use of animals.

Open Access Statement: This is an Open Access article distributed in accordance with the Creative Commons Attribution-NonCommercial-NoDerivs 4.0 International License (CC BY-NC-ND 4.0), which permits the noncommercial replication and distribution of the article with the strict proviso that no changes or edits are made and the original work is properly cited (including links to both the formal publication through the relevant DOI and the license). See: https://creativecommons.org/licenses/by-nc-nd/4.0/.

\section{References}

1. Brunham RC, Gottlieb SL, Paavonen J. Pelvic inflammatory disease. N Engl J Med 2015;372:2039-48.

2. Ross J, Guaschino S, Cusini M, et al. 2017 European guideline for the management of pelvic inflammatory disease. Int J STD AIDS 2018;29:108-14.

3. French CE, Hughes G, Nicholson A, et al. Estimation of the rate of pelvic inflammatory disease diagnoses: trends in England, 2000-2008. Sex Transm Dis 2011;38:158-62.

4. Risser WL, Risser JM, Risser AL. Current perspectives in the USA on the diagnosis and treatment of pelvic inflammatory disease in adolescents. Adolesc Health Med 
Ther 2017;8:87-94.

5. Das BB, Ronda J, Trent M. Pelvic inflammatory disease: improving awareness, prevention, and treatment. Infect Drug Resist 2016;9:191-7.

6. Yeh JM, Hook EW 3rd, Goldie SJ. A refined estimate of the average lifetime cost of pelvic inflammatory disease. Sex Transm Dis 2003;30:369-78.

7. Sheldon IM, Rycroft AN, Dogan B, et al. Specific strains of Escherichia coli are pathogenic for the endometrium of cattle and cause pelvic inflammatory disease in cattle and mice. PLoS One 2010;5:e9192.

8. Zhou Z, Zeng F, Yuan J, et al. Pelvic inflammatory disease and the risk of ovarian cancer: a meta-analysis. Cancer Causes Control 2017;28:415-28.

9. Zhang X, He M, Lei S, et al. An integrative investigation of the therapeutic mechanism of Ainsliaea fragrans Champ. in cervicitis using liquid chromatography tandem mass spectrometry based on a rat plasma metabolomics strategy. J Pharm Biomed Anal 2018;156:221-31.

10. Oh Y, Lee J, Kim HC, et al. Establishment of hydrochloric acid/lipopolysaccharide-induced pelvic inflammatory disease model. J Vet Sci 2016;17:413-9.

11. Tang B, Wu K, Meng Q, et al. Comparison of the Analgesic and Anti-Inflammatory Effects of Xiaoyuningkun Decoction with Cynanchum Paniculatum and Fukeqianjin in a Mouse Model of Pelvic Inflammatory Disease. Med Sci Monit 2019;25:9094-102.

12. Wei A, Feng H, Jia XM, et al. Ozone therapy ameliorates inflammation and endometrial injury in rats with pelvic inflammatory disease. Biomed Pharmacother 2018;107:1418-25.

13. Tsevat DG, Wiesenfeld HC, Parks C, et al. Sexually transmitted diseases and infertility. Am J Obstet Gynecol 2017;216:1-9.

14. Reekie J, Donovan B, Guy R, et al. Risk of Pelvic Inflammatory Disease in Relation to Chlamydia and Gonorrhea Testing, Repeat Testing, and Positivity: A Population-Based Cohort Study. Clin Infect Dis 2018;66:437-43.

15. Mitchell C, Prabhu M. Pelvic inflammatory disease: current concepts in pathogenesis, diagnosis and treatment. Infect Dis Clin North Am 2013;27:793-809.

16. Moreno I, Cicinelli E, Garcia-Grau I, et al. The diagnosis of chronic endometritis in infertile asymptomatic women: a comparative study of histology, microbial cultures, hysteroscopy, and molecular microbiology. Am J Obstet Gynecol 2018;218:602.e1-602.e16.

17. Ness RB, Kip KE, Hillier SL, et al. A cluster analysis of bacterial vaginosis-associated microflora and pelvic inflammatory disease. Am J Epidemiol 2005;162:585-90.

18. Curry A, Williams T, Penny ML. Pelvic Inflammatory Disease: Diagnosis, Management, and Prevention. Am Fam Physician 2019;100:357-64.

19. Kak G, Raza M, Tiwari BK. Interferon-gamma (IFN- $\gamma$ ): Exploring its implications in infectious diseases. Biomol Concepts 2018;9:64-79.

20. Gieseck RL 3rd, Wilson MS, Wynn TA. Type 2 immunity in tissue repair and fibrosis. Nat Rev Immunol 2018;18:62-76.

21. Chen KS, Wang PH, Yang SF, et al. Significant elevation of a Th2 cytokine, interleukin-10, in pelvic inflammatory disease. Clin Chem Lab Med 2008;46:1609-16.

22. Paludan SR. Interleukin-4 and interferon-gamma: the quintessence of a mutual antagonistic relationship. Scand J Immunol 1998;48:459-68.

23. Nguyen JK, Austin E, Huang A, et al. The IL-4/IL-13 axis in skin fibrosis and scarring: mechanistic concepts and therapeutic targets. Arch Dermatol Res 2020;312:81-92.

24. Akhurst RJ, Hata A. Targeting the TGF $\beta$ signalling pathway in disease. Nat Rev Drug Discov 2012;11:790-811.

25. Jin X, Ren S, Macarak E, et al. Pathobiological mechanisms of peritoneal adhesions: The mesenchymal transition of rat peritoneal mesothelial cells induced by TGF- $\beta 1$ and IL- 6 requires activation of Erk1/2 and Smad2 linker region phosphorylation. Matrix Biol 2016;51:55-64.

26. Abudukeyoumu A, Li MQ, Xie F. Transforming growth factor- $\beta 1$ in intrauterine adhesion. Am J Reprod Immunol 2020;84:e13262.

27. Salma U, Xue M, Ali Sheikh MS, et al. Role of Transforming Growth Factor- $\beta 1$ and Smads Signaling Pathway in Intrauterine Adhesion. Mediators Inflamm 2016;2016:4158287.

28. Li J, Du S, Sheng X, et al. MicroRNA-29b Inhibits Endometrial Fibrosis by Regulating the Sp1-TGF- $\beta 1 /$ Smad-CTGF Axis in a Rat Model. Reprod Sci 2016;23:386-94.

(English Language Editors: A. Kassem and J. Gray)

Cite this article as: Fan L, Liu Z, Zhang Z, Li T, Li H, Chen J, Zong X, Zhang X, Chen X, Bai H, Wang F, Shang C. Identifying the clinical presentations, progression, and sequela of pelvic inflammatory disease through physiological, histological and ultrastructural evaluation of a rat animal model. Ann Trans Med 2021;9(23):1710. doi: 10.21037/atm-21-3345 